PROCEEDINGS OF THE

AMERICAN MATHEMATICAL SOCIETY

Volume 135, Number 5, May 2007, Pages 1441-1447

S 0002-9939(06)08636-9

Article electronically published on November 13, 2006

\title{
GENUS 2 MAPPING CLASS GROUPS ARE NOT KÄHLER
}

\author{
RĂZVAN VELICHE \\ (Communicated by Michael Stillman) \\ To Oana and " $A A A$ "
}

\begin{abstract}
The goal of this note is to prove that the mapping class groups of closed orientable surfaces of genus 2 (with punctures) are not Kähler. An application to compactifications of the moduli space of genus $g$ curves (with punctures) is given.
\end{abstract}

\section{INTRODUCTION}

It is a long-standing problem to determine which groups can occur in the class $\mathcal{K}$ of fundamental groups of compact Kähler manifolds. There are many examples and positive results, but there are also many restrictions (negative tests) that prohibit groups from being in $\mathcal{K}$ (see $\mathrm{ABC}: 96$ ).

Related to the class $\mathcal{K}$, one can introduce several other classes of fundamental groups: $\mathcal{Q K}$ (of complements of divisors in Kähler manifolds), $\mathcal{P}$ (of projective varieties or manifolds) and $\mathcal{Q P}$ (of quasi-projective varieties). Since every projective manifold is a Kähler one, we have

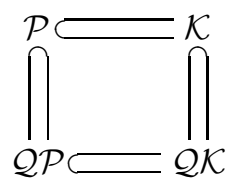

(and it is an open question whether the horizontal inclusions are equalities).

The mapping class group $M(g, n)$ of (punctured) closed orientable surfaces is in $\mathcal{Q P}(2.10)$. One may ask the question of whether this group is in $\mathcal{P}$. In this note a negative answer is given in the genus 2 case.

An immediate consequence is that $\mathcal{M}_{2, n}$, the moduli space of $n$-punctured curves of genus 2, cannot have a projective compactification with a "thinner" boundary (i.e. of higher codimension): the Satake compactification has a codimension two component "at infinity", and the Deligne-Mumford compactification has a divisor there.

\section{NOTATIONS AND BACKGROUND}

2.1. In what follows, a variety is an irreducible but possibly non-reduced scheme over $\mathbb{C}$. A manifold is a smooth variety (i.e. it is reduced, too).

Received by the editors February 25, 2005 and, in revised form, December 16, 2005.

2000 Mathematics Subject Classification. Primary 32G15. (C)2006 American Mathematical Society
Reverts to public domain 28 years from publication 1441 
2.2. For a compact orientable (connected) topological manifold $T$ one defines the (pure) braid group $=P_{0, n}(T)$ as the fundamental group of

$$
\boldsymbol{F}_{0, n}(T)=\left\{\left(z_{1}, \ldots, z_{n}\right) \in T^{n} \mid z_{i} \neq z_{j}, \forall 1 \leq i \neq j \leq n\right\} .
$$

There is a free action (by permutation) of the symmetric group $S_{n}$ on $\boldsymbol{F}_{0, n}(T)$, and one denotes the fundamental group of the quotient manifold $\boldsymbol{B}_{0, n}(T)=\boldsymbol{F}_{0, n}(T) / S_{n}$ by $\bar{P}_{0, n}(T)$ (called the full braid group).

2.3. Introducing punctures, one defines $P_{k, n}(T)$ as $\pi_{1}\left(\boldsymbol{F}_{0, n}\left(T \backslash Q_{k}\right)\right)$, where $Q_{k}$ is any set of distinct $k$ points in $T$ (its choice does not matter, as one can always find a homeomorphism of $T$ mapping one choice onto another), and respectively $\bar{P}_{k, n}(T)$ as the fundamental group of $\boldsymbol{B}_{k, n}(T)=\boldsymbol{F}_{k, n}(T) / S_{n}$.

2.4. It is known that $P_{k, n}(T)$ and $B_{k, n}(T)$ are finitely generated groups; see e.g. Bir75, §1.2,1.3].

2.5. It is easy to see ([Bir75, Prop.1.1]) that there are extensions

$$
1 \rightarrow P_{k, n}(T) \rightarrow \bar{P}_{k, n}(T) \rightarrow S_{n} \rightarrow 1 .
$$

2.6. Related to these, one can consider $\mathcal{F}_{n}(T)$ as the space of orientation-preserving homeomorphisms of $T$ fixing a given $n$-tuple $\left(z_{1}^{0}, \ldots, z_{n}^{0}\right)$ of distinct points of $T$, and $\mathcal{B}_{n}(T)$ as the space of orientation-preserving homeomorphisms of $T$ fixing the set $\left\{z_{1}^{0}, \ldots, z_{n}^{0}\right\}$. Both of these spaces are endowed with the compact-open topology. Denote by $M^{p}(T, n)=\pi_{0}\left(\mathcal{F}_{n}(T)\right)$ (the pure mapping class group of $T$ ) and by $M(T, n)=\pi_{0}\left(\mathcal{B}_{n}(T)\right)$ the (full) mapping class group of $T$ (also denoted in the literature by $\Gamma_{g, n}$ ). These are the groups of isotopy classes of (pointwise, respectively setwise) puncture-preserving diffeomorphisms.

2.7. As in the case of the braid groups, we have the extensions

$$
1 \rightarrow M^{p}(T, n) \rightarrow M(T, n) \rightarrow S_{n} \rightarrow 1 .
$$

2.8. In the case when $T$ is a closed oriented surface of genus $g, M(T, n)$ is classically denoted by $M(g, n)([\operatorname{Bir} 75])$ or $M_{g}^{n}$.

2.9. This note is concerned with the structure of $M(g, n)$, and particularly with the possibility of it being the fundamental group of a Kähler manifold.

2.10. Note that $M(g, n)$ is the fundamental group of a quasi-projective manifold. Namely, the Teichmüller space $\mathcal{T}_{g, n}$ of equivalence classes of orientation- (and puncture-) preserving diffeomorphisms of a genus $g$ smooth curve, is a contractible complex manifold. The mapping class group $M(g, n)$ acts properly discontinuously on $\mathcal{T}_{g, n}$, but the action is not necessarily free; actually, even the pure mapping class (sub)group $M^{p}(g, n)$ might act on $\mathcal{T}_{g, n}$ with fixed points (this does not happen as soon as $n>2 g+2)$. However, the quotient $\mathcal{M}$ has at most finite-quotient singularities (it is an orbifold); $M(g, n)$ becomes its orbifold fundamental group $\pi_{1}^{\text {orb }}(\mathcal{M})$, and there exists a finite index subgroup $H$ (which can be chosen to be normal) of $M(g, n)$ which acts freely on $\mathcal{T}_{g, n}$ ([" Hai00, 12.8]), and such that $H \backslash \mathcal{T}_{g, n}$ is quasiprojective and smooth. That means that there is a finite (orbifold) cover $\widetilde{\mathcal{M}} \rightarrow \mathcal{M}$ with $\widetilde{\mathcal{M}}$ a smooth quasi-projective manifold. Now one may use the argument in ABC:96, Lemma 1.15, p. 7] to show that $M(g, n)$ is the fundamental group of a (smooth) quasi-projective manifold. 
2.11. We recall the definition of commensurability: it is the equivalence relation in the category of groups generated by morphisms with finite kernel and cokernel.

2.12. We will be using some results on the ends of a (Kähler) group, so it is worth saying a few words on the subject.

For a topological space $X$, the set of ends of $X$ is the inverse $\operatorname{limit} \lim \pi_{0}(X \backslash K)$, where the limit is taken as $K$ ranges over all compact subsets of $X$. The cardinality of this set is denoted by $e(X)$. If a group $G$ acts freely on a connected simplicial complex $X$ with finite quotient, then $e(X)$ is independent of $X$, and is denoted by $e(G)$; this is the number of ends of the group $G$.

2.13. Facts (see Coh72, SW79]): A free group with more than two generators has infinitely many ends. The number of ends of an infinite group can be 1,2 or $\infty$. Commensurable groups have the same number of ends.

2.14. A finite index subgroup of a group in $\mathcal{K}$ (or $\mathcal{P}$ ) is also in $\mathcal{K}$ (resp. $\mathcal{P}$ ); this is roughly due to the possibility of lifting and averaging a Kähler metric to a finite unramified cover, respectively to the fact that the pullback of an ample divisor to a finite cover is ample.

However, a finite extension of a group in $\mathcal{K}$ need not be in $\mathcal{K}$; e.g. if $G \cong \mathbb{Z}^{2} \rtimes \mathbb{Z}_{2}$ by the action switching the generators of $\mathbb{Z}^{2}$, then $G$ is not in $\mathcal{K}$ (the first Betti number is 1).

\section{Preliminaries}

We start by a minor generalization of a result in [Bir75, (4.1, p. 151)].

Lemma 3.1. The evaluation map $\epsilon_{g, n, k}: \mathcal{F}_{k}(T) \rightarrow F_{k, n}(T)$ is a locally trivial fibering with fibre $\mathcal{F}_{n+k}(T)$.

Proof. The argument is quasi-identical to the proof in [Bir75, but we include it here for the reader's convenience.

Fix an $(n+k)$-tuple $\left(z_{1}^{0}, \ldots, z_{k+n}^{0}\right)$ of distinct points in $T$. Without restricting the generality, the homeomorphisms in $\mathcal{F}_{i}(M)$ can be taken to fix $\left(z_{1}^{0}, \ldots, z_{i}^{0}\right)$ (for $i \in\{k, n+k\}) . \mathcal{F}_{n+k}(T)$ then becomes a closed topological subgroup of $\mathcal{F}_{k}(T)$.

The evaluation map $\epsilon_{g, n, k}$ takes a homeomorphism $h$ to $\left(h\left(z_{k+1}^{0}\right), \ldots, h\left(z_{n+k}^{0}\right)\right)$, which is clearly a point in $F_{k, n}(T)$ thought of as $F_{0, n}\left(T \backslash\left\{z_{1}^{0}, \ldots, z_{k}^{0}\right\}\right)$.

$\epsilon_{g, k, n}$ is surjective (any finite set of points can be taken to another set of the same cardinality by a homeomorphism of $T$ ), and two homeomorphisms $h_{1}$ and $h_{2}$ have the same image under $\epsilon_{g, k, n}$ if and only if they are in the same left coset of $\mathcal{F}_{n+k}(T)$ in $\mathcal{F}_{k}(T)$.

Thus $F_{k, n}(T)$ is naturally (topologically) identified with the quotient space $\mathcal{F}_{k}(T) / \mathcal{F}_{n+k}(T)$, and $\epsilon_{g, k, n}$ becomes the projection map which turns $F_{k, n}(T)$ into a homogeneous space.

By [Hu59, p. 99], the theorem follows as soon as we show the existence of a local section for $\epsilon_{g, k, n}$ near $\mathbf{z}^{0}=\epsilon_{g, k, n}\left(\mathcal{F}_{n+k}(T)\right)$.

For that, choose pairwise disjoint Euclidean neighbourhoods $U\left(z_{k+1}^{0}\right), \ldots$, $U\left(z_{n+k}^{0}\right)$ of $z_{k+1}^{0}, \ldots, z_{k+n}^{0}$; we require that the union of these neighbourhoods does not intersect $\left\{z_{1}^{0}, \ldots, z_{k}^{0}\right\}$.

Then $U\left(\mathbf{z}^{0}\right)=\left\{\left(u_{k+1}, \ldots, u_{k+n}\right) \mid u_{i} \in U\left(z_{i}^{0}\right)\right\}$ is a neighbourhood of $\mathbf{z}^{0}$ in $F_{k, n}(T)$. Construct a family of homeomorphisms $\left\{f_{u} \in \mathcal{F}_{k}(T) \mid u \in U\left(\mathbf{z}^{0}\right)\right\}$ depending continuously on $u$ and such that for each $u \in U\left(\mathbf{u}^{0}\right)$ : 
- $f_{u}\left(z_{k+j}^{0}\right)=u_{k+j}(j=\overline{1, n})$ and

- $f_{u}\left(T \backslash \underset{j=k+1}{\bigcup_{u}+n} U\left(z_{j}^{0}\right)\right)$ is the identity.

Such a family exists because, for example, there exists a continuous family of homeomorphisms (even diffeomorphisms) deforming the center of a ball to any of the points in the ball.

Now the section we wanted is given by $s(u)=f_{u}$.

3.2. In the case of a closed oriented surface of genus $g$, the lemma gives, via the long exact sequence in homotopy associated to a fibration,

$$
P_{k, n}\left(T_{g}\right) \rightarrow M^{p}(g, n+k) \rightarrow M^{p}(g, k) \rightarrow \pi_{0}\left(F_{k, n}(T)\right)=1 .
$$

3.3. We will make essential use of the fact that there is an exact sequence

$$
1 \rightarrow \mathbb{Z}_{2} \rightarrow M(2,0) \rightarrow M(0,6) \rightarrow 1
$$

(see [Bir75, p. 188]).

3.4. In [Bir75, Thm. 4.2, p. 152] it is proven that there is an exact sequence

$$
1 \rightarrow P_{0, n}\left(T_{g}\right) \rightarrow M^{p}(g, n) \rightarrow M^{p}(g, 0) \rightarrow 1
$$

for $g \geq 2$, and a similar one

$$
1 \rightarrow P_{0, n}\left(T_{g}\right) / \text { center } \rightarrow M^{p}(g, n) \rightarrow M^{p}(g, 0) \rightarrow 1
$$

if $g=1, n \geq 2$ or $g=0, n \geq 3$.

3.5. In Bir75, Thm 1.4, p. 14] the existence of the following exact sequence is proven:

$$
1 \rightarrow P_{n-1,1}(M) \rightarrow P_{0, n}(M) \rightarrow P_{0, n-1}(M) \rightarrow 1
$$

for any $n \geq 1$. One needs $M$ to satisfy $\pi_{i}\left(M \backslash Q_{m}\right)=1$ for $i=0,2,3$ and for every $m \geq 0$ as in the hypotheses. In the case of $S^{2}$ (which fails the above test for $m=0$ ) the exact sequence is valid only for $n \geq 4$ (see [Bir75, p. 34]).

3.6. We will need the fact that $P_{0,3}\left(S^{2}\right)=\mathbb{Z}_{2}$; see [Bir75, p. 34].

\section{MAin Result}

Theorem 4.1. The groups $M(2, n)$ are not Kähler, for any $n \geq 0$.

Proof. The idea of the proof is rather simple: show that these groups have too "big" an image to be Kähler.

More precisely, ABR92 proves that an extension of a group with infinitely many ends by a finitely generated group cannot be Kähler.

Thus, if we prove that inside each of the $M(2, n)$ 's there is a finite index subgroup mapping (with finitely generated kernel) onto a group with infinitely many ends, we are done.

The natural subgroup of finite index to consider in each $M(g, n)$ is $M^{p}(g, n)$ (2.7). The exact sequence (3.4) for $g=2$ and the remark (2.4) show that it is enough to prove that $M^{p}(2,0)=M(2,0)$ maps (with finitely generated kernel) onto a group with infinitely many ends.

Now (3.3) reduces the problem to $M(0,6)$. This does not map directly to what we need, but its (finite index) subgroup $M^{p}(0,6)$ maps, by (3.2), onto $M^{p}(0,4)$ with finitely generated kernel. 
Now the second sequence in (3.4) with $g=0$ and $n=4$ gives $M^{p}(0,4) \cong$ $P_{0,4}\left(S^{2}\right) /$ center.

But (3.5) for $n=4$ (and $g=0$ ) gives:

$$
1 \rightarrow P_{3,1}\left(S^{2}\right) \rightarrow P_{0,4}\left(S^{2}\right) \rightarrow P_{0,3}\left(S^{2}\right) \rightarrow 1 \text {. }
$$

But $P_{0,3}\left(S^{2}\right)=\mathbb{Z}_{2}(\underline{3.6})$, and $P_{3,1}\left(S^{2}\right)=\pi_{1}\left(S^{2} \backslash\left\{z_{1}^{0}, z_{2}^{0}, z_{3}^{0}\right\}\right) \cong \pi_{1}(\mathbb{C} \backslash 0,1)=\mathbb{F}_{2}$ (the free group on two generators). Now it is easy to see that the center of $P_{0,4}\left(S^{2}\right)$ is either trivial or isomorphic to $\mathbb{Z}_{2}$ (it is actually the latter, see Bir75, Lemma 4.2 .3 , p.154]), and since it cannot intersect the $\mathbb{F}_{2}$, one has a finite index copy of $\mathbb{F}_{2}$ inside $M^{p}(0,4)$ (we actually have $M^{p}(0,4) \cong \mathbb{F}_{2}$ ). Pulling back this copy of $\mathbb{F}_{2}$ to either $M^{p}(0,6)$ or $M^{p}(2,0)$ finishes the proof.

\section{Higher genus; CONJECTURE}

5.1. The exact sequence (3.2) for compact surfaces of genus $g \geq 3, n=1$ and $k \geq 1$ yields

$$
P_{k, 1}\left(T_{g}\right) \stackrel{d}{\rightarrow} M^{p}(g, k+1) \stackrel{i}{\rightarrow} M^{p}(g, k) \rightarrow 1,
$$

where $d$ is the "boundary" map $\pi_{1}\left(F_{1,1}\left(T_{g}\right)\right) \rightarrow \pi_{0}\left(\mathcal{F}_{2}\left(T_{g}\right)\right)$ and $i$ is induced by the fibre-inclusion $\mathcal{F}_{2}\left(T_{g}\right) \rightarrow \mathcal{F}_{1}\left(T_{g}\right)$.

Lemma 5.2. $\operatorname{ker}(d) \subseteq \operatorname{center}\left(P_{k, 1}\left(T_{g}\right)\right)$.

Proof. Easy adaptation of the proof of [Bir75, Lemma 4.2.1, p. 153].

Since $P_{k, 1}\left(T_{g}\right)=\pi_{1}\left(T_{g} \backslash\left\{z_{1}, \ldots, z_{k}\right\}\right)=\mathbb{F}_{2 g+k-1}$, the lemma gives the existence of a finitely generated free normal subgroup of infinite index in $M^{p}(g, k+1)$.

5.3. At this point, we would like to introduce the class NNF of groups that do not have normal free finitely generated subgroups (of rank at least 2).

\section{Conjecture 5.4. $\mathcal{K} \subset \mathrm{NNF}$.}

Remark 5.5. The validity of this conjecture would imply that all the mapping class groups $M(g, n)$ with $g \geq 2$ and $n \geq 2$ are not in $\mathcal{K}$.

Remark 5.6. It is known that any (finitely generated) free group has a finite index normal subgroup of odd rank. Then (2.14) shows that groups in $\mathcal{K}$ cannot have finite index normal free subgroups (these would be finitely generated, actually).

Thus the conjecture refers to the infinite index case.

5.7. As support for this conjecture we cite the work of [Cat03], showing that the fundamental groups of surfaces of genus $g$ are of class NNI. That is, a more restrictive class, introduced by Catanese, where no finitely generated normal subgroups (not necessarily free) of infinite index are allowed in the given group.

NNI $\nsubseteq \mathbf{N N F}$ (take for example a direct product of a free group, of rank at least 2 , with $\mathbb{Z}_{2}$ ). But if one enlarges NNF to NNIF (prohibiting only infinite index finitely generated normal free subgroups, of rank at least 2), one has NNI $\subset$ NNIF.

Note that we have $\mathcal{K} \cap \mathbf{N N I} \subseteq \mathcal{K} \cap \mathbf{N N I F}=\mathcal{K} \cap \mathbf{N N F}$ (the latter by (2.14)). The conjecture simply says that $\mathcal{K} \subset$ NNIF.

Remark 5.8. The classes NNI, NNIF, $\mathcal{K} \cap \mathbf{N N I}$ and $\mathcal{K} \cap \mathbf{N N I F}$ are not closed under direct products. It is an easy observation that $\mathbf{N N F}$ and $\mathcal{K} \cap \mathbf{N N F}$ are closed under this operation. This implies that arbitrary (finite) direct products of finite groups and fundamental groups of smooth projective curves are in $\mathcal{K} \cap \mathbf{N N F}$. 
Remark 5.9. As proved in [Coh72, Prop. 2.5, p. 23], if a group $G$ has a subgroup $H$ which is not locally finite, but which is included in a finitely generated subgroup of infinite index, then $G$ has one end. In particular a direct product of two free groups of rank greater than 2 has only one end. So the obstruction in (and a proof of) the above conjecture should have more geometric content.

\section{Relevance to compactifications of $\mathcal{M}_{g, n}$}

6.1. A natural question that arises in connnection to $\mathcal{M}_{g, n}$ is whether one could find a projective (more generally, a weakly Kählerian) compactification for it, such that the "boundary" has high codimension. The Deligne-Mumford compactification has a divisor, and the Satake compactification has a codimension two component in the boundary.

Proposition 6.2. $\mathcal{M}_{2, n}$ has no projective compactification with boundary of codimension at least 3 .

Using 4.1, 2.10 and 2.14, this is an immediate consequence of the following.

Lemma 6.3. Given:

(1) a quasi-projective orbifold $X$ with an irreducible universal cover $\widetilde{X}$,

(2) a (normal) finite index subgroup $H$ of $\pi_{1}^{\text {orb }}(X)$ such that $Y=H \backslash \widetilde{X}$ is quasi-projective and smooth, and such that

(3) $H \notin \mathcal{P}$,

then $X$ cannot have a projective compactification with boundary of codimension at least 3 .

Proof. Assume there would be such a compactification, denoted by $\bar{X} \hookrightarrow \mathbb{P}^{N}$. The map $p: Y \rightarrow X$ is onto and finite by hypothesis. Composition with the inclusion $X \hookrightarrow \bar{X}$ gives a dominant quasi-finite map $f: Y \rightarrow \bar{X} \subseteq \mathbb{P}^{N}$.

Since the codimension of the boundary $\bar{X} \backslash X$ is at least 3 and $\bar{X}$ is projective, we can find an irreducible subscheme $L^{\prime}=L \cap \bar{X}$ in $\bar{X}$, of dimension 2, with $L$ a linear space of $\mathbb{P}^{N}$, and such that $L^{\prime} \cap(\bar{X} \backslash X)=\emptyset$. This implies that for a sufficiently small $\epsilon$ (and a Riemannian metric on $\mathbb{P}^{N}$ ), the neighbourhood $L_{\epsilon}^{\prime}$ of points at distance less than $\epsilon$ from $L$ is also avoiding the boundary $\bar{X} \backslash X$.

Now we can use the theorem of Goresky and MacPherson (GM83]; see also [FL81, §9]) regarding pullbacks of linear subspaces of $\mathbb{P}^{N}$ via quasi-finite morphisms, to get that $\pi_{i}\left(Y, f^{-1}\left(L_{\epsilon}^{\prime}\right)\right)=1$ for $i \leq 2$.

This implies $\pi_{1}(Y)=\pi_{1}\left(f^{-1}\left(L_{\epsilon}^{\prime}\right)\right)=\pi_{1}\left(f^{-1}\left(L^{\prime}\right)\right)$.

Now $f^{-1}\left(L^{\prime}\right)=p^{-1}\left(L^{\prime}\right) \rightarrow L^{\prime}$ is a finite map, and $L^{\prime}$ is projective. Then $p^{-1}\left(L^{\prime}\right)$ is also projective.

But then $\pi_{1}(Y)=H$ would be in $\mathcal{P}$, a contradiction.

Note: it is sufficient to assume in the lemma that $Y$ is a (connected, but possibly reduced) local complete intersection (see [FL81, §9]).

\section{REFERENCES}

[ABC:96] J. Amoros, M. Burger, K. Corlette, D. Kotschick, and D. Toledo. Fundamental Groups of Compact Kähler Manifolds, volume 44 of Mathematical Surveys and Monographs. American Mathematical Society, 1996. MR1379330 (97d:32037)

[ABR92] D. Arapura, P. Bressler, and M. Ramachandran. On the fundamental group of a compact kähler manifold. Duke Math. J., 68(3):477-488, 1992. MR1194951 (94e:57040) 
[Bir75] Joan S. Birman. Braids and Links and and Mapping Class Groups, volume 82 of Annals of Mathematics Studies. Princeton University Press, 1975. MR0375281 (51:11477)

[Cat03] Fabrizio Catanese. Fibred Kähler and quasi-projective groups. Adv. Geom. 2003, suppl., S13-S27. MR2028385 (2004m:32034)

[Coh72] D.E. Cohen. Groups of Cohomological Dimension One, volume 245 of Lecture Notes in Math. Springer-Verlag, Berlin, 1972. MR0344359 (49:9098)

[FL81] W. Fulton and R. Lazarsfeld. Connectivity and its applications in algebraic geometry. In Algebraic Geometry (Chicago, Ill., 1980), volume 862 of Lecture Notes in Math. Springer, Berlin, New York, 1981. MR0644817 (83i:14002)

[GM83] M. Goresky and R. MacPherson. Stratified Morse theory. In Singularities, Part 1 (Arcata, Calif., 1981), volume 40 of Proc. Sympos. Pure Math. Amer. Math. Soc., Providence, R.I., 1983. MR0713089 (84k:58017)

[Hai00] Richard Hain. Moduli of Riemann surfaces, transcendental aspects. In School on Algebraic Geometry (Trieste, 1999), volume 1 of ICTP Lect. Notes. Abdus Salam Int. Cent. Theoret. Phys., Trieste, 2000. MR1795866 (2001m:32030)

[Hu59] Sze-Tsen Hu. Homotopy Theory. Academic Press, New York, 1959. MR0106454 $(21: 5186)$

[SW79] G.P. Scott and C.T.C. Wall. Topological methods in group theory. In Homological Group Theory, volume 36 of London Math. Soc. Lecture Note Ser. Cambridge Univ. Press, Cambridge, 1979. MR0564422(81m:57002)

Department of Mathematics, Purdue University, West Lafayette, Indiana 47907

Current address: Department of Mathematics, University of Utah, Salt Lake City, Utah 84112

E-mail address: rveliche@math.utah.edu 